
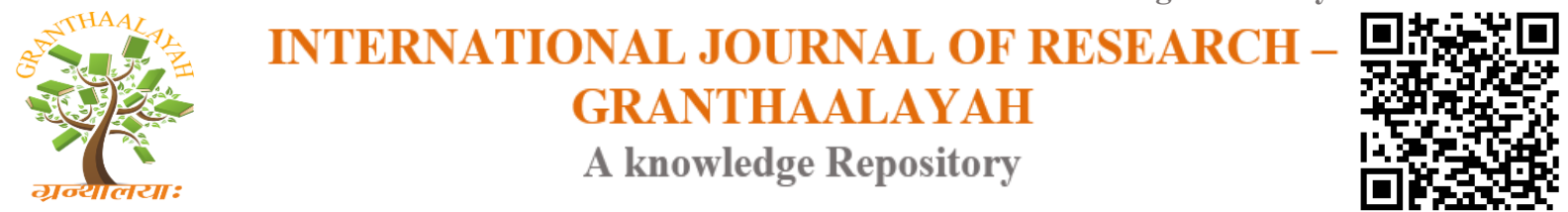

Science

\title{
OPTIMAL CONTROL ANALYSIS OF THE DYNAMICAL SPREAD OF MEASLES
}

\author{
S. O. Adewale ${ }^{* 1}$, I. A. Olopade ${ }^{2}$, S. O. Ajao ${ }^{3}$, G. A. Adeniran ${ }^{4}$ \\ ${ }^{*} 1,3,4$ Department of Pure and Applied Mathematics, Ladoke Akintola University of Technology \\ (LAUTECH), Ogbomoso, NIGERIA \\ 2 Department of Mathematics and Computer Science, Elizade University, Ilara-Mokin, \\ NIGERIA
}

\begin{abstract}
In this paper, a five (5) compartmental model is presented to study the transmission dynamics of Measles in a population at any point in time. The model is rigorously analyzed to gain insight into the dynamical features of Measles and also, optimal control theory is applied to give an optimality system which we used to minimize the number of infected individuals and propose the most suitable control strategy for the spread of measles. It is shown that the model has a diseases free equilibrium which is globally asymptotically stable (GAS). Also, there exists a unique endemic equilibrium point which is locally stable whenever the associated threshold quantity exceeds (one) unity. We also show that there exists a solution for the optimality system. From the result, it was observed that vaccine control strategy is more efficient in reducing the number of infected individuals as compared to other control strategies.
\end{abstract}

Keywords:

Measles, Reproduction number, Optimal Control, Vaccination, Isolation, Epidemic.

Cite This Article: S. O. Adewale, I. A. Olopade, S. O. Ajao, and G. A. Adeniran, "OPTIMAL CONTROL ANALYSIS OF THE DYNAMICAL SPREAD OF MEASLES" International Journal of Research - Granthaalayah, Vol. 4, No. 5 (2016): 169-188.

\section{INTRODUCTION}

Measles is a communicable and deadly viral disease caused by the measles virus, of the genus Morbillivirus which belongs to the family paramyxoviridae. This disease can be contacted on a close contact with an infected individual via airborne propagules. When an infected individual sneezes or coughs, measles is spread through droplet transmission from the nose, throat, and mouth of someone who is infected with Measles virus. The infection leads to the development of a typical rash /fever illnesses. Measles is preventable by vaccine. Information has it that if unvaccinated (unimmunized) people exposed to the virus, more than $90 \%$ will contract the disease. Measles is highly contagious, when the virus droplets are released into the air, the virus 
can still remain in the air (and still able to cause infections) for up to two hours after an infected person has left a room. Once one had measles and recovered from it, then the person is believed to be immune for life against the disease. Also, people who have received two doses of vaccine after their first birthday have a $98 \%$ likelihood of being immune (that is the probability of having measles even when in contact with those infected with measles is about 0.02 , which is very low). Children when born, receive some forms of immunity from their mother, but unfortunately, this immunity is not complete, and therefore infants are at increased risk of infection until they receive the vaccination at 12 to 15 months of age $[1,2,3,27,30]$.

The typical time from exposure to a person infected with measles to development of the initial symptoms is 10-12 days (the range is 7 to 21 days). The rash occurs a few days after the initial symptoms (ranges from 7 to 18 days from exposure). The period of infection spans through one week and, after which the hosts recover and develop lifelong immunity. Therefore, people can only have measles infection once in their lifetime. If the force of infection that is the effective contact rate is sufficiently large, high incidence rate would be recorded mostly among the young age, and hence measles is a childhood disease. When most people in a community at any point in time are not vaccinated, measles outbreak is imminent which poses serious danger $[1,2,25,26,27,30]$.

Children under the age of one are the most vulnerable to measles (although they have some immunity passed from their mother, which is not $100 \%$ effective and wane easily); also, people who have not received the proper vaccination series; people who received immunoglobulin at the time of measles vaccination and people who had taken outdated, ineffective measles vaccine. Malnutrition, concurrent infection, and inadequate case management are the major factors responsible for some high mortality rates of measles especially in the tropical Africa. In this region, we have heard of about 5\%,10\% and some worst situation of $20 \%$ reported cases. Infection with measles leads to complications in one out of seven cases and is very severe in about one out of five thousands cases in developed countries, where there is no adequate case management, $[1,17,20,21,25,26,30]$.

Disease prevention is key to public health. It is always better to prevent a disease than to treat it. Unfortunately, in the case of measles there is no specific drug for the treatment of the diseases. Though, some physician recommends the use of some pain reliever, giving of enough vitamin A and humidification of the air as some of forms of treatment to measles. Vaccines can protect both the people who receive them and those with whom they come in contact. Vaccines are responsible for the control of many infectious diseases that were once common in so many countries around the world, which includes polio, measles, diphtheria, pertussis (whooping cough), rubella (German measles), mumps, tetanus, and Haemophilus influenzae type b (Hib). Over the years, vaccines have prevented countless cases of infectious diseases and saved literally millions of lives [20,21].

Also, people most likely to have complications (including death) are those who are malnourished or who have weakened immune systems. In most people, the disease produces fever (temperature $>101 \mathrm{~F}[38.3 \mathrm{C}]$ ), a generalized rash that last greater than three days, cough, runny nose (coryza), and red eyes (conjunctivitis). The complications of measles that result in most deaths include pneumonia and inflammation of the brain (encephalitis). Any woman that contract 
measles during pregnancy period may have a miscarriage, a stillbirth, or a preterm delivery. There appears to be no risk of having birth defects (unlike an infection with the rubella virus, known as German measles) [1,6, 27,30].

Measles is a highly contagious viral disease that has high mortality rate within few days. Although global incidence has been significantly reduced through vaccination, measles remains an important public health problem. Measles, the leading vaccine-preventable killer of children worldwide, is estimated to have caused 614000 global deaths annually in 2002, with 50\% of all global measles deaths occurring in sub-Saharan Africa. The persistence of measles in many African countries points to the need to further investigate the dynamics of measles epidemics in endemic region [12,15]. Similarly, in 2008, measles killed 164,000 children worldwide. However, measles is rarely fatal in the United States. Research shows that this is due to the fact that most people are immunized, which results in very infrequent outbreaks. On the other hands, Measles cases are increasing in Ireland, with 320 cases reported between August 2009 and early 2010 , and 206 cases were due to the unvaccinated [29,30].

Despite the enormous efforts by the World Health Organization (WHO) and United Nations Children's Fund (UNICEF) at reducing the global burden of measles, it has remained a public health challenge. Worldwide, measles is fifth leading cause of death among under-five children with an estimated 197,000 deaths in 2007 [21,28]. Measles infection is still prevalent in many developing countries especially in parts of Africa and Asia where more than 20 million measles cases are reported annually [30]. In the West Africa sub-region, large and recurrent epidemics associated with high mortality have occurred in Niger (2003), Nigeria (2004) and Chad (2004) [6]. Frequent epidemics continue to occur in countries that have not fully implemented the WHO strategy [6,21,25]. In 2010, the Asia continent tops the global list of measles cases with Malawi having 118,712 cases followed by China $(38,159)$ cases and India $(29,808)$ cases. The second in rank by continent is Europe with Bulgaria leading the continent with 22,004 cases. France was ranked eleventh in the world with 5,048 cases and United Kingdom having 443 cases reported. In Africa, Zambia top the list with 15,754 cases followed by Zimbabwe and Nigeria with 9,696 and 8,491 cases respectively. In America, Canada has 99 reported cases and was ranked 63 in the global record for measles in that year. Similarly, Australia which was ranked 70 , has 70 cases reported for the year 2010 [31]. While in 2011, there were 222 cases of measles confirmed in the United States. [26].

The more children in a community that are vaccinated, the less likely it is that any children, even those who have not been immunized, will get sick because there are fewer hosts for the infectious agents. This is referred to as "herd" immunity and it is particularly vital with extremely contagious diseases such as measles, where immunization of 90 to 95 per cent of infants is needed to protect a community from measles epidemics. However, this is not true for all diseases, such as tetanus, therefore an individual's vaccination status is important, not just group immunity [7,12,23,29]. Vaccine-preventable diseases have a costly impact, resulting in doctor's visits, hospitalizations, and premature deaths. Sick children can also cause parents to lose time from work [8].

Many researchers have investigated the nature, characteristics, effect and modes of spread of Measles in the societies; these researches are either experimental or theoretical. Also, many 
mathematical models have been used to gain insight into the transmission dynamics and control of Measles spread in human population. The purpose of the current study is to provide a rigorous optimality control analysis of a model for the spread of Measles. The model uses standard incidence function for the infection rate and some time dependent control variables to obtain an optimal system shall be introduce so as to obtain the most efficient control strategy.

The paper is organized as follows. The model is formulated in Section 2, and is qualitatively analyzed in Section 3. In Section 4, we introduced some time dependent control variables and obtained the optimality system. Section 5, we carried out the numerical simulations while section 6 is the discussion of all the results of both the mathematical analysis and the numerical simulations.

\section{MODEL FORMULATION}

Following $[3,15,17,18]$, the total homogeneously-mixing population at time $t$, denoted by $\mathrm{N}(\mathrm{t})$, is sub divided into mutually-exclusive compartments of susceptible $(\mathrm{S}(\mathrm{t}))$, exposed/latent $(\mathrm{E}(\mathrm{t}))$, infectious $(\mathrm{I}(\mathrm{t}))$, isolated infectious $(\mathrm{J}(\mathrm{t}))$ individual and recovered $(\mathrm{R}(\mathrm{t}))$ individuals, so that $\mathrm{N}(\mathrm{t})=\mathrm{S}(\mathrm{t})+\mathrm{E}(\mathrm{t})+\mathrm{I}(\mathrm{t})+\mathrm{J}(\mathrm{t})+\mathrm{R}(\mathrm{t}):$

The susceptible population is increased by the recruitment of people (either by birth or immigration) into the population (all recruited individuals are assumed to be susceptible), at a rate $\pi$. Also, the susceptible population increases by the recovered individuals. Anyone who has had measles is believed to be immune for life. People who have received two doses of vaccine after their first birthday have a $98 \%$ likelihood of being immune. This population is decreased by infection, which can be acquired following effective contact with infectious individuals only, at a rate $\lambda$ given by

$$
\lambda=\frac{\beta\left(\eta_{d} I(t)+\eta_{j} J(t)\right)}{N(t)}
$$

In (1), $\beta$ represents the effective contact rate (i.e., contact capable of leading to measles infection), $\eta_{d}$ is a modification parameter that compares the transmissibility of the disease. Here, we also assume that $0<\eta_{d} \leq 1$. Similarly, $0<\eta_{j} \leq 1, \eta_{j}$ is modification parameter that accounts for the reduced transmissibility of infectious individuals in the isolated class. Finally, this population decreases by natural death (at a rate $\mu$ ). Thus, the rate of change of the susceptible population is given by

$$
\frac{d S(t)}{d t}=\pi+\sigma R(t)-\lambda S(t)-\mu S(t)
$$

The population of the exposed individuals increases when individuals from the susceptible class are in contact with individuals with measles but have not been showing the symptoms of the diseases. The population of exposed individuals is decreased by the progression of exposed individuals to infectious measles stage i.e a stage when individuals has started showing symptoms of the diseases (at a rate $\kappa$ ). It is pertinent to mention here that $\kappa$ depends greatly on the level of individuals immunity which has been acquire mostly during birth via series of 
immunization program. Again, the population of the exposed individuals is further reduced by natural death (at the rate $\mu$ ). Thus,

$$
\frac{d E(t)}{d t}=\lambda S(t)-(\kappa+\mu) E(t)
$$

The population of the infectious individuals is increased by Progression of the exposed individuals into an active infectious stage (at the rate $\kappa$ ). This population is decreased (at a rate $\gamma$ ), when infected individuals are treated and recovered from the disease. Also, the population of the infectious individuals are decreased by natural death (at the rate $\mu$ ), disease-induced mortality (at a rate $\delta$ ) and isolation (at the rate $\xi$ ). Hence,

$$
\frac{d I(t)}{d t}=\kappa E(t)-\left(\xi+\gamma_{1}+\mu+\delta\right) I(t)
$$

We also assume that among those infectious individuals (those that reported at the hospital for treatment), some were isolated for proper monitoring of treatment. So, the population of the isolated infectious individuals is increased by Progression of the infectious individuals into isolated infectious class (at the rate $\xi$ ). This population is decreased (at a rate $\gamma_{2}$ ), when isolated infected individuals are treated and recovered from the disease. Also, the population in this class are decreased by natural death (at the rate $\mu$ ) and disease-induced mortality (at a rate $\left.\delta_{j}\right)$. Hence,

$$
\frac{d J(t)}{d t}=\xi I(t)-\left(\gamma_{2}+\mu+\delta_{j}\right) J(t)
$$

Recovery here means recovery from the disease. The population of recovered individuals is increased by the recovery of infectious individuals and isolated infectious individuals after treatment. This population is decreased by removing those who have recovered from the diseases to the susceptible category since individuals who recovered from measles diseases is assumed to have permanent immunity to the diseases because from the literature, individuals can only have the diseases once in life time. Again, the recovered individuals population decreased by natural death (at the rate $\mu$ ).

Therefore,

$$
\frac{d R(t)}{d t}=\gamma_{1} I(t)+\gamma_{2} J(t)-\sigma R-\mu R
$$

Thus, in summary, the measles dynamics transmission model, is given by the following system of non-linear differential equations,

$$
\begin{aligned}
& \frac{d S(t)}{d t}=\pi+\sigma R(t)-\lambda S(t)-\mu S(t) \\
& \frac{d E(t)}{d t}=\lambda S(t)-(\kappa+\mu) E(t) \\
& \frac{d I(t)}{d t}=\kappa E(t)-\left(\xi-\gamma_{1}+\mu+\delta\right) I(t) \\
& \frac{d J(t)}{d t}=\xi I(t)-\left(\gamma_{2}+\mu+\delta_{j}\right) J(t) \\
& \frac{d R(t)}{d t}=\gamma_{1} I(t)+\gamma_{2} J(t)-\sigma R(t)-\mu R(t)
\end{aligned}
$$




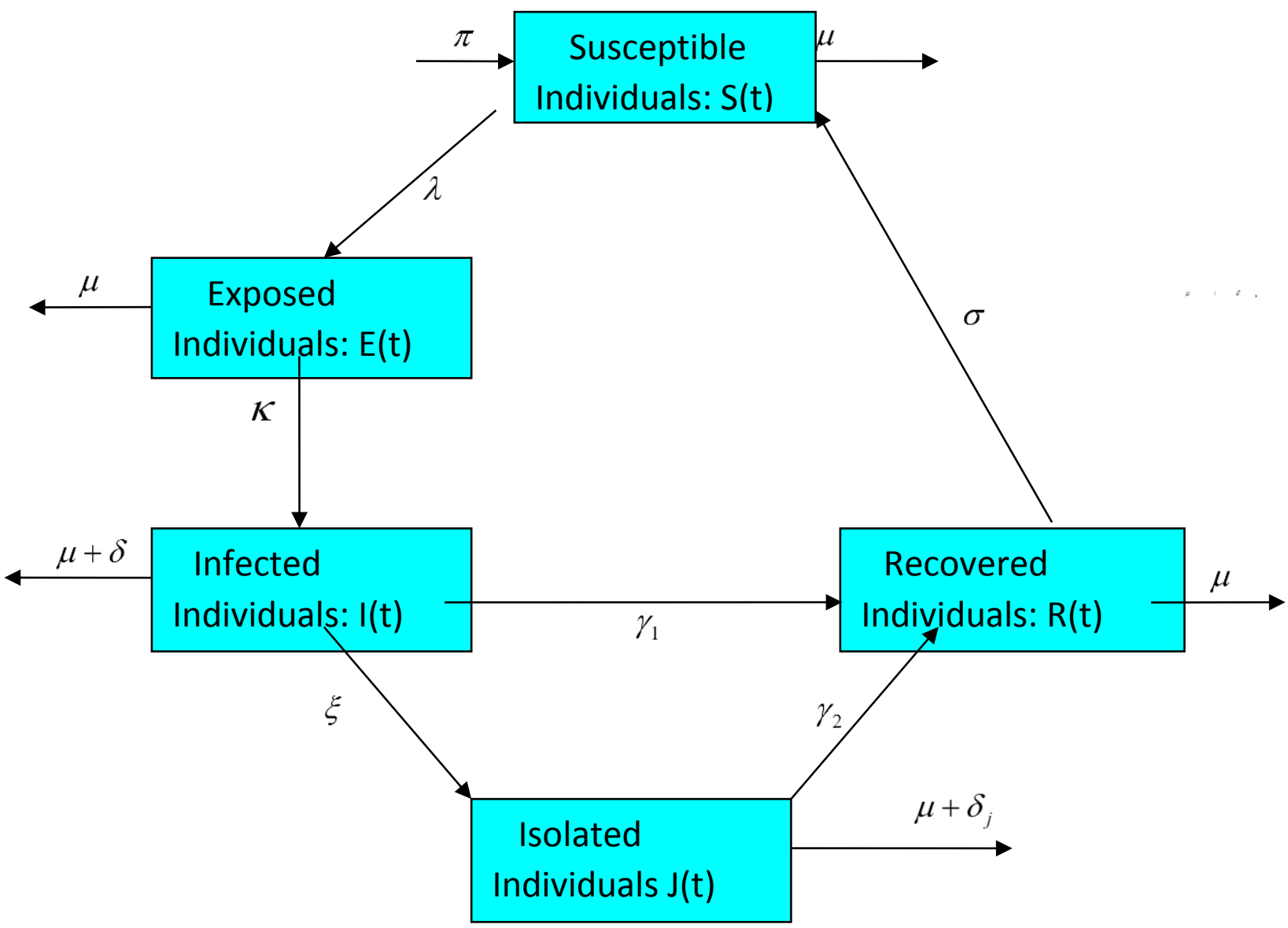

Figure 1: Schematic diagram of the model (2.7)

Table 1: Description of parameters of the model (2.7).

\begin{tabular}{ll}
\hline Parameters & Descriptions \\
\hline$\beta$ & Effective contact rate for Measles infection \\
$\mu$ & Per capita natural mortality rate \\
$\Pi$ & Recruitment rate into the population \\
$\kappa$ & Progression rate to infectious class \\
$\gamma_{1}, \gamma_{2}$ & Treatment rate for infectious and isolated individuals respectively \\
$\delta, \delta_{j}$ & Measles induced mortality rate for Infected class and Isolated infected \\
$\sigma$ & class \\
$\eta_{d} \eta_{j}$ & Recovery rate for individuals \\
$\xi$ & Modification parameters \\
\hline
\end{tabular}




\section{ANALYSIS OF THE MODEL}

Theorem 3.1: The closed set $D=\left\{(S, E, I, J, R) \in \mathfrak{R}_{+}^{5}: N \leq \frac{\pi}{\mu}\right\}$ is positively-invariant and attracting with respect to the model equations (2.7).

Proof:: Consider the biologically-feasible region, D, defined above. The rate of change of the total population, obtained by adding all the equations of the model (2.7), is given by

$\frac{d N}{d t}=\pi-\mu S-\mu E-\mu I-\mu J-\mu R-\delta I-\delta_{j} J$

And since $N(t)=S(t)+E(t)+I(t)+J(t)+R(t) ;(2.8)$ implies that

$\frac{d N(t)}{d t}=\pi-\mu N(t)-\delta I(t)-\delta_{j} J(t)$

Therefore, $\frac{d N(t)}{d t}<0$ whenever the sub total population $N(t)>\frac{\pi}{\mu}$ Hence, for all time $t>0$, all the solutions of the model with the initial conditions in $D$ will remain in $D$. Thus, the biologically feasible region $D$ is positively- invariant and attracting. This completes the proof. In the region $\mathrm{D}$, the model can be considered as being epidemiologically and mathematically well-posed $[2,3,4,16]$.

\subsection{Disease-free equilibrium (DFE)}

The model (2.7) has a DFE, obtained by setting the right-hand sides of the equations of the model to zero, given by

$$
\varepsilon_{0}=\left(S_{0}, E_{0}, I_{0}, J_{0}, R_{0}\right)=\left\{\frac{\pi}{\mu}, 0,0,0,0\right\}
$$

The stability of the DFE, $\varepsilon_{0}$, will be analyzed using the next generation method (see [2,9]). The non-negative matrix $F$ (of the new infection terms) and the non-singular matrix $V$ (of the remaining transfer terms) are given, respectively, by

$F=\left(\begin{array}{cccc}0 & \beta \eta_{d} & \beta \eta_{j} & 0 \\ 0 & 0 & 0 & 0 \\ 0 & 0 & 0 & 0 \\ 0 & 0 & 0 & 0\end{array}\right)$

$V=\left(\begin{array}{lccc}K_{1} & 0 & 0 & 0 \\ -\kappa & K_{2} & 0 & 0 \\ 0 & -\xi & K_{3} & 0 \\ 0 & -\gamma_{1} & -\gamma_{2} & K_{4}\end{array}\right)$

Where $K_{1}=(\kappa+\mu), \quad K_{2}=\left(\xi+\gamma_{1}+\mu+\delta\right) \quad K_{3}=\left(\gamma_{2}+\mu+\delta_{j}\right) \quad$ and $K_{3}=(\sigma+\mu)$ 
The associated reproduction number, denoted by $R_{0}$ is given by $R_{0}=\rho\left(F V^{-1}\right)$, where $\rho$ denotes the spectral radius (dominant eigenvalue in magnititude) of the next generation matrix $\left(F V^{-1}\right)$.

It follows that

$R_{0}=\frac{\beta \kappa\left(\eta_{d} K_{3}+\eta_{j} \xi\right)}{K_{1} K_{2} K_{3}}$

Hence, the result below follows from Theorem 2 of [9]

Lemma 3.1. The DFE of the model equation (2.7), given by (3.3), is locally stable if $R_{0}<1$, and unstable if $R_{0}>1$. The threshold quantity, $R_{0}$, is the reproduction number for the model. It measures the average number of new Measles infections generated by a single infectious individual in a population where some of the infected individuals have been immunized. The epidemiological implication of Lemma 3.1 is that Measles spread can be effectively controlled in the community (when $R_{0}<1$ ) if the initial sizes of the sub- populations of the model are in the basin of attraction of the disease-free equilibrium $\varepsilon_{0}=\left(\frac{\pi}{\mu}, 0,0,0,0\right)$

Epidemiologically, if $R_{0}<1$, the disease will dies out in the community and if $R_{0}>1$, the disease spreads in the population. Hence, the basic Reproduction numbers turned out to be an important factor in determining the transmission dynamics of any infectious diseases.

\subsection{Stability Analysis of the DFE}

Here, the stability property of the DFE of model (2.7) will be explored. At a steady-state, $S(t)=N(t)^{*}-E(t)-I(t)-J(t)-R(t)$, hence the stability of $\varepsilon_{0}$ can be established by considering the following mass action equivalent of the model (2.7) given as;

$$
\begin{aligned}
& \frac{d E(t)}{d t}=\lambda\left(N(t)^{*}-E(t)-I(t)-J(t)-R(t)\right)-(\kappa+\mu) E(t) \\
& \frac{d I(t)}{d t}=\kappa E(t)-\left(\xi+\gamma_{1}+\mu+\delta\right) I(t) \\
& \frac{d J(t)}{d t}=\xi I(t)-\left(\gamma_{2}+\mu+\delta_{j}\right) J(t) \\
& \frac{d R(t)}{d t}=\gamma_{1} I(t)+\gamma_{2} J(t)-\sigma R(t)-\mu R(t)
\end{aligned}
$$

And $\lambda=\frac{\beta\left(\eta_{d} I(t)+\eta_{j} J(t)\right)}{N(t)}$

Here, the invariance region is given by

$$
D^{*}=\left\{(E(t), I(t), J(t), R(t)) \in \mathfrak{R}_{+}^{4}: E(t)+I(t)+R(t) \leq N(t)^{*}\right\}
$$

Theeorem 3.2: The DFE of the model (3.6), given by (3.3) is Global Asymptotically Stable (GAS) if $R_{0}<1$. 
Proof: The equations (3.6) can be re-written as

$\left(\begin{array}{l}\frac{d E}{d t} \\ \frac{d I}{d t} \\ \frac{d J}{d t} \\ \frac{d R}{d t}\end{array}\right)=\left(G_{1}-G_{2}-G_{3}\right)\left(\begin{array}{l}E \\ I \\ J \\ R\end{array}\right)$

Where the matrices $G_{1}, G_{2}$ and $G_{3}$ are given by;

$G_{1}=\left(\begin{array}{cccc}0 & \beta \eta_{d} & \beta \eta_{j} & 0 \\ 0 & 0 & 0 & 0 \\ 0 & 0 & 0 & 0 \\ 0 & 0 & 0 & 0\end{array}\right) \quad G_{2}=\left(\begin{array}{cccc}K_{1} & 0 & 0 & 0 \\ -\kappa & \left(\xi+\gamma_{1}+\mu+\delta\right) & 0 & 0 \\ 0 & -\xi & \left(\gamma_{2}+\mu+\delta_{j}\right) & 0 \\ 0 & -\gamma_{1} & -\gamma_{2} & (\sigma+\mu)\end{array}\right)$

And

$$
G_{3}=\left(\begin{array}{cccc}
\lambda & \lambda & \lambda & \lambda \\
0 & 0 & 0 & 0 \\
0 & 0 & 0 & 0 \\
0 & 0 & 0 & 0
\end{array}\right)
$$

Since matrix $G_{3}$ is non-negative thus,

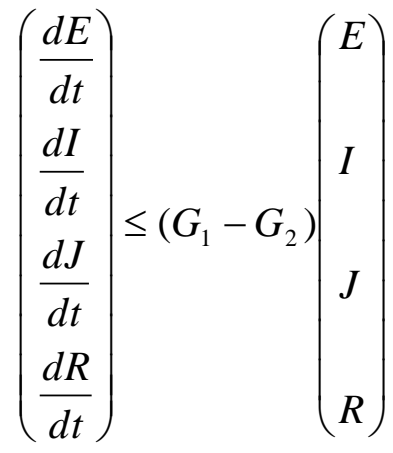

If $R_{0}<1$ then $\ell\left(G_{1} G_{2}^{-1}\right)<1$ (from the local stability result given in lemma 3.1), which is equivalent to $G_{1}-G_{2}$ having all its eigenvalues in the left-half plane [20]. It follows that the linearized differential inequality system (3.6) is stable whenever $R_{0}<1$. Consequently, by comparison theorem [20], it follows that $(E(t), I(t), J(t), R(T) \rightarrow(0,0,0,0)$. Hence, since $D$ is positively-invariant, it follows that DFE is GAS in $D^{*}$ if $R_{0}<1$.

\subsection{Existence of endemic equilibrium point (EEP)}

In this section, the possible existence and stability of endemic (positive) equilibria of the model (2.7) (i.e., equilibria where at least one of the infected components of the model is non-zero) will be considered. 
Let $\varepsilon_{1}=\left(S^{*}, E^{*}, I^{*}, J^{*}, R^{*}\right)$ represents any arbitrary endemic equilibrium of the model equation (2.7). Solving the equations of the system at the steady-state gives,

$$
\begin{aligned}
S^{*} & =\frac{\pi+\sigma R^{*}}{\lambda^{*}+\mu} \\
E^{*} & =\frac{\lambda^{*} S^{*}}{(\kappa+\mu)} \\
I^{*} & =\frac{\kappa E^{*}}{\left(\xi+\gamma_{1}+\mu+\delta\right)} \\
J^{*} & =\frac{\xi I^{*}}{\left(\gamma_{2}+\mu+\delta_{j}\right)} \\
R^{*} & =\frac{\gamma_{1} I+\gamma_{2} J^{*}}{(\sigma+\mu)}
\end{aligned}
$$

The expression for $\lambda$ defined in (3.10) above, at the endemic steady-state, denoted by

$$
\lambda=\frac{\beta\left(\eta_{d} I(t)+\eta_{j} J(t)\right)}{N(t)}
$$

We re-write this in terms of $\lambda^{*} S^{*}$ as follows;

$$
\begin{aligned}
& E^{*}=\frac{\lambda^{*} S^{*}}{K_{1}} \\
& I^{*}=\frac{\kappa \lambda^{*} S^{*}}{K_{1} K_{2}}=P_{1} \lambda^{*} S^{*} \\
& J^{*}=\frac{\xi \kappa \lambda^{*} S^{*}}{K_{1} K_{2} K_{3}}=P_{2} \lambda^{*} S^{*} \\
& R^{*}=\frac{\gamma_{1} \kappa \lambda^{*} S^{*}}{K_{1} K_{2} K_{4}}+\frac{\gamma_{2} \xi \kappa \lambda^{*} S^{*}}{K_{1} K_{2} K_{3} K_{4}}=P_{3} \lambda^{*} S^{*}
\end{aligned}
$$

Where, $K_{1}=(\kappa+\mu), \quad K_{2}=\left(\xi+\gamma_{1}+\mu+\delta\right) \quad K_{3}=\left(\gamma_{2}+\mu+\delta_{j}\right) \quad$ and $K_{3}=(\sigma+\mu)$ and

$$
\begin{aligned}
& P_{1}=\frac{\kappa}{K_{1} K_{2}} \\
& P_{2}=\frac{\xi \kappa}{K_{1} K_{2} K_{3}} \\
& P_{3}=\frac{\gamma_{1} \kappa}{K_{1} K_{2} K_{4}}+\frac{\gamma_{2} \xi \kappa}{K_{1} K_{2} K_{3} K_{4}}
\end{aligned}
$$

Substituting the expressions in (3.12) with (3.13) into (3.11) gives

$$
\lambda^{*}\left(S^{*}+\frac{\lambda^{*} S^{*}}{K_{1}}+P_{1} \lambda^{*} S^{*}+P_{2} \lambda^{*} S^{*}+P_{3} \lambda^{*} S^{*}\right)=\beta \lambda^{*} S^{*}\left(\eta_{d} P_{1}+\eta_{j} P_{2}\right)
$$

Dividing each term in (3.14) by $\lambda^{*} S^{*}$ (and noting that at endemic steady-state $\lambda^{*} S^{*} \neq 0$ ) gives 
$1+P_{4} \lambda^{*}=\beta\left(\eta_{d} P_{1}+\eta_{j} P_{2}\right)$

Where $P_{4}=\frac{1}{K_{1}}+P_{1}+P_{2}+P_{3} \succ 0$.

Thus, $1+P_{4} \lambda^{*}=\frac{\beta \kappa\left(\eta_{d} K_{3}+\eta_{j} \xi\right)}{K_{1} K_{2} K_{3}}=R_{0}^{*}$

Hence,

$\lambda^{*}=\frac{R_{0}{ }^{*}-1}{P_{4}}>0$

Whenever $R_{0}>1$.

The components of the unique endemic equilibrium $\left(\varepsilon_{1}\right)$ can then be obtained by substituting the unique value of $\lambda^{*}$, given in (3.15), into the expressions in (3.10). Thus, the following result has been established.

\subsection{Local stability of EEP}

The local stability of the unique EEP, $\varepsilon_{1}$, will now be explored for the special case where the disease-induced mortality is negligible (i.e. $\delta=\delta_{j}=0$ ), Setting $\delta=\delta_{j}=0$ in the model (2.7) shows that

$\frac{d N(t)}{d t}=\pi-\mu N(t)$

Hence, it follows from (3.16) that $N(t) \rightarrow \frac{\pi}{\mu}=N^{*}$ as $t \rightarrow \infty$ : Further, using the substitution $S=N^{*}-E-I-R$ (and noting that $\delta=\delta_{j}=0$ ) in the model equation (2.7) gives the following reduced model

$$
\begin{aligned}
\frac{d E(t)}{d t} & =\frac{\beta\left(\eta_{d} I(t)+\eta_{j} J(t)\right)\left(N(t)^{*}-E(t)-I(t)-J(t)-R(t)\right)}{N(t)^{*}}-(\kappa+\mu) E(t) \\
\frac{d I(t)}{d t} & =\kappa E(t)-\left(\xi+\gamma_{1}+\mu\right) I(t) \\
\frac{d J(t)}{d t} & =\xi I(t)-\left(\gamma_{2}+\mu\right) J(t) \\
\frac{d R(t)}{d t} & =\gamma_{1} I(t)+\gamma_{2} J(t)-\sigma R(t)-\mu R(t)
\end{aligned}
$$

So for the reduced model (3.15), the associated reproduction number denoted by $R^{*}{ }_{0}$ is given by

$$
R_{0}^{*}=\frac{\beta \kappa\left(\eta_{d} K_{3}+\eta_{j} \xi\right)}{K_{1} K_{2} K_{3}}
$$

Using the same approach as in section 3.3, it can be shown that the reduced model equation (3.17) has a unique endemic equilibrium given by $\varepsilon_{1} \mid \delta=\delta_{j}=0$ whenever $R_{0}^{*}>1$. 
The epidemiological implication of this is that once there is endemic situation of measles in a community, the diseases will persist in that community whenever the associated reproduction number $R_{0}^{*}>1$. Hence to avoid the endemic situation we should find a means of keeping the associated reproduction number $R^{*}{ }_{0}$ less than unity.

\section{MATHEMATICAL ANALYSIS OF OPTIMALITY OF THE SYSTEM}

In this section, we provide the mathematical analysis of the possible control strategy that will help the public health practitioners achieved the best strategy in the control of the spread of measles in the society having in mind the condition obtained in the previous section, that is there will be an endemic situation whenever the reproduction number is greater than unity. In order to derive the necessary conditions for these optimal control variables, we now introduce time dependent preventive measures $\left(u_{1}, u_{2}\right)$ and treatment $\left(u_{3}\right)$ efforts as control strategy for the spread of measles. So the model equation (2.7) becomes:

$$
\begin{aligned}
& \frac{d S(t)}{d t}=\pi+\sigma R(t)-\left(1-u_{1}\right) \lambda S(t)-\mu S(t) \\
& \frac{d E(t)}{d t}=\left(1-u_{1}\right) \lambda S(t)-(\kappa+\mu) E(t) \\
& \frac{d I(t)}{d t}=\kappa E(t)-(\xi+\mu+\delta) I(t)-\left\{\left(\left(1-u_{2}\right)+\left(1-u_{3}\right)\right\} I(t)\right. \\
& \frac{d J(t)}{d t}=\xi I(t)-\left(\mu+\delta_{j}\right) J(t)-\left(1-u_{3}\right) J(t) \\
& \frac{d R(t)}{d t}=\left(1-u_{3}\right) I(t)+\left(1-u_{3}\right) J(t)-\sigma R(t)-\mu R(t)
\end{aligned}
$$

Let the function $0 \leq u_{1} \leq 1$ denote the control strategy for the use of vaccine as at time due, while $0 \leq u_{2} \leq 1$ represents the effectiveness of educating the society of the menace of measles. Again, we let $0 \leq u_{3} \leq \alpha,(0 \leq \alpha \leq 1)$ represent the control on treatment where $\alpha$ is the drug efficacy used for the treatment. Since treatments cannot be continued infinitely over period of time because of the negative side effect, so for our control classes we choose measurable functions which is defined on a fixed interval that satisfy $0 \leq a_{i} \leq u_{i}(t) \leq b_{i} \prec 1$ for $\mathrm{i}=1,2,3$. All other parameters is as defined in the model (2.1) and (2.7).

\subsection{Existence of an Optimal Control Pair}

Following the results of Fleming and Rishel in[10] and in Hattsf and Yousfi [12,13], we obtained the existence of an optimal control pair for the model (4.1).

Theorem 4.1:Given the control model (4.1), then there exists an optimal control pair $\underset{u_{i}}{\longrightarrow}=\left(u_{1}^{*}, u_{2}{ }^{*}, u_{3}^{*}\right) \in U$ such that $J\left(u_{1}^{*}, u_{2}{ }^{*}, u_{3}{ }^{*}\right)=\underset{u_{1}^{*}, u_{2}^{*}, u_{3}^{*}}{\operatorname{Max}}\left\{J\left(u_{1}, u_{2}, u_{3}\right)\right\}$

Proof: To use an existence results in [10], we need to verify the following properties of the model;

(1) The set of controls and corresponding state variables is nonempty

(2) The control U set is convex and closed 
(3) The right hand side of the state system is bounded by a linear function in the state and control variables

(4) The integrand of the objective functional is concave on $U$

(5) There exist constants $P_{1}, P_{2} \succ 0$ and $\eta \succ 1$ such that the integrand $L\left(I, u_{1}, u_{2}, u_{3}\right)$ of the objective functional satisfies $L\left(I, u_{1}, u_{2}, u_{3}\right) \leq P_{2}-P_{1}\left(\left|u_{1}\right|^{2}+\left|u_{2}\right|^{2}+\left|u_{3}\right|^{2}\right)^{\frac{\eta}{2}}$

To check if the conditions above are satisfies, we use the result by Hattaf and Yousfi $[12,13]$ and Lukes [18] to give the existence of solutions of the model (4.1) with bounded coefficients which gives the first condition. We note that the solution are bounded and by definition, the control set $\left(u_{1}, u_{2}, u_{3}\right)$ is convex and closed, therefore condition 2 is satisfies. We note that the solution are bounded and positive and since our state system is bilinear in $u_{1}, u_{2}, u_{3}$, hence the right hand side of model (4.1) satisfy condition 3. Also, we note that the integrand of our objective functional is concave, hence the fourth condition is easily verified. Also we have the last condition needed $L\left(I, u_{1}, u_{2}, u_{3}\right) \leq P_{2}-P_{1}\left(\left|u_{1}\right|^{2}+\left|u_{2}\right|^{2}+\left|u_{3}\right|^{2}\right)$

Where $P_{2}$ depends on the upper bound on I and $P_{1}>0$ since $A_{1}, A_{2}, A_{3}>0$. Since all these five conditions have been verify to hold, we therefore conclude that there exists an optimal control pair. This completes the proof.

\subsection{Optimality System}

The Objective functional to be minimized is given as:

$$
J\left(u_{1}, u_{2}, u_{3}\right)=\int_{0}^{t_{f}}\left(a I+A_{1} u_{1}^{2}+A_{2} u_{2}^{2}+A_{3} u_{3}^{2}\right) d t
$$

Here the constants $a, A_{1}, A_{2}, A_{3}$ are all positive weights to balance the size of the terms. $t_{f}$ is the final time of interest while zero(0) is the initial time. The objective here is to minimize the number of infectious individuals $\mathrm{I}(\mathrm{t})$, while minimizing the cost of control $\left.u_{1}(t), u_{2} t\right), u_{3}(t)$, . We therefore seek optimal control pair $u_{1}{ }^{*}, u_{2}{ }^{*}, u_{3}{ }^{*}$, such that:

$$
J\left(u_{1}^{*}, u_{2}^{*}, u_{3}^{*}\right)=\underset{u_{1}^{*}, u_{2}^{*}, u_{3}^{*}}{{ }^{*}}\left\{J\left(u_{1}, u_{2}, u_{3}\right) /\left(u_{1}, u_{2}, u_{3}\right) \in U\right\}
$$

Where $U=\left\{\left(u_{1}, u_{2}, u_{3}\right) / u_{i}\right.$ is measurable, $a_{i} \leq u_{i} \leq b_{i}, t \in\left[0, t_{f}\right] \rightarrow[0,1]$ for $\left.i=1,2,3\right\}$ is the control set.

The term aI is the cost of infection while $A_{1} u_{1}{ }^{2}, A_{2} u_{2}{ }^{2}, A_{3} u_{3}{ }^{2}$ are the costs of vaccination at the due time, education/ campaign awareness and treatment efforts respectively. Now, we obtain the optimal control pair using Pontryagin's maximum principle [24]. This principle converts equations (4.2) and (4.3) into a problem of minimizing pointwise a Hamiltonian $\mathrm{H}$ with respect to $\left(u_{1}, u_{2}, u_{3}\right)$. So we have: 


$$
\begin{aligned}
& H=a I+A_{1} u_{1}^{2}+A_{2} u_{2}^{2}+A_{3} u_{3}^{2}+\phi_{S}\left[\pi+\sigma R(t)-\left(1-u_{1}\right) \lambda S(t)-\mu S(t)\right] \\
& +\phi_{E}\left[\left(1-u_{1}\right) \lambda S(t)-(\kappa+\mu) E(t)\right] \\
& +\phi_{I}\left[\kappa E(t)-(\xi+\mu+\delta) I(t)-\left\{\left(\left(1-u_{2}\right)+\left(1-u_{3}\right)\right\} I(t)\right]\right. \\
& +\phi_{J}\left[\xi I(t)-\left(\mu+\delta_{j}\right) J(t)-\left(1-u_{3}\right) J(t)\right] \\
& +\phi_{R}\left[\left(1-u_{3}\right) I(t)+\left(1-u_{3}\right) J(t)-\sigma R(t)-\mu R(t)\right]
\end{aligned}
$$

Theorem 4.2: Given an optimal control $u_{1}{ }^{*}, u_{2}{ }^{*}, u_{3}{ }^{*}$, and solutions $S^{*}, E^{*}, I^{*}, J^{*}, R^{*}$ of the corresponding state system (4.1) that minimizes the objective functional $J\left(u_{1}, u_{2}, u_{3}\right)$ over $\mathrm{U}$, there exists adjoint variables $\phi_{S}, \phi_{E}, \phi_{I}, \phi_{J}, \phi_{R}$ satisfying

$$
\begin{aligned}
-\frac{d \phi_{S}}{d t}= & -\phi_{S}\left[\left(1-u_{1}\right) \lambda-\mu\right]+\left(1-u_{1}\right) \lambda \phi_{E} \\
-\frac{d \phi_{E}}{d t}= & -(\kappa+\mu) \phi_{E}+\kappa \phi_{I} \\
-\frac{d \phi_{I}}{d t}= & a-\left[\left(1-u_{1}\right) \beta \eta_{d} S(t) \phi_{S}-\left(1-u_{1}\right) \beta \eta_{d} S(t) \phi_{E}\right]+(\xi+\mu+\delta) \phi_{I} \\
& -\left[\left(1-u_{2}\right)+\left(1-u_{3}\right)\right] \phi_{I}+\xi \phi_{J}+\left(1-u_{3}\right) \phi_{R} \\
-\frac{d \phi_{J}}{d t}= & -\left[\left(1-u_{1}\right) \beta \eta_{j} S(t) \phi_{S}-\left(1-u_{1}\right) \beta \eta_{j} S(t) \phi_{E}\right]-\phi_{J}\left[\left(\mu+\delta_{j}\right)+\left(1-u_{3}\right)\right] J(t)+\left(1-u_{3}\right) \phi_{R} \\
-\frac{d \phi_{R}}{d t}= & \sigma \phi_{S}-(\sigma+\mu) \phi_{R} \\
\phi_{S}\left(t_{f}\right)= & \phi_{E}\left(t_{f}\right)=\phi_{I}\left(t_{f}\right)=\phi_{J}\left(t_{f}\right)=\phi_{R}\left(t_{f}\right)=0,
\end{aligned}
$$

tranversality conditions with the controls $u_{1}{ }^{*}, u_{2}{ }^{*}$, and $u_{3}{ }^{*}$ satisfying the optimality condition;

$$
\begin{aligned}
& u_{1}^{*}=\max \left\{0, \min \left(1, \frac{\lambda S^{*}(t)\left[\phi_{E}-\phi_{S}\right]}{2 A_{1}}\right)\right\} \\
& u_{2}^{*}=\max \left\{0, \min \left(1, \frac{-I^{*}(t) \phi_{I}}{2 A_{2}}\right)\right\} \\
& u_{3}^{*}=\max \left\{0, \min \left(1, \frac{\left(I^{*}(t)+J^{*}(t)\right) \phi_{R}-\left(I^{*}(t) \phi_{I}+J^{*}(t) \phi_{J}\right)}{2 A_{3}}\right)\right\}
\end{aligned}
$$

Proof: Following Pontryagin's maximum principle, we obtained the standard form of the adjoint equations and tranversality conditions by differentiating the Hamiltonian function with respect to states $\mathrm{S}, \mathrm{E}, \mathrm{I}, \mathrm{J}$, and R respectively which is evaluated at the optimal control function $u_{1}, u_{2}, u_{3}$ So we re-write the adjoint system as follow: 


$$
\begin{aligned}
-\frac{d \phi_{S}}{d t}= & \frac{\partial H}{\partial S}=-\phi_{S}\left[\left(1-u_{1}\right) \lambda-\mu\right]+\left(1-u_{1}\right) \lambda \phi_{E} \\
-\frac{d \phi_{E}}{d t}= & \frac{\partial H}{\partial E}=-(\kappa+\mu) \phi_{E}+\kappa \phi_{I} \\
-\frac{d \phi_{I}}{d t}= & \frac{\partial H}{\partial I}=a-\left[\left(1-u_{1}\right) \beta \eta_{d} S(t) \phi_{S}-\left(1-u_{1}\right) \beta \eta_{d} S(t) \phi_{E}\right]+(\xi+\mu+\delta) \phi_{I} \\
-\frac{d \phi_{J}}{d t}= & -\left[\left(1-u_{2}\right)+\left(1-u_{3}\right)\right] \phi_{I}+\xi \phi_{J}+\left(1-u_{3}\right) \phi_{R} \\
-\frac{d \phi_{R}}{d t}= & \left.-\frac{\partial H}{\partial R}=\sigma\left(1-u_{1}\right) \beta \eta_{j} S(t) \phi_{S}-\left(1-u_{1}\right) \beta \eta_{j} S(t) \phi_{E}\right]-\phi_{J}\left[\left(\mu+\delta_{j}\right)+\left(1-u_{3}\right)\right] J(t)+\left(1-u_{3}\right) \phi_{R}
\end{aligned}
$$

With tranversality conditions $\phi_{S}\left(t_{f}\right)=\phi_{E}\left(t_{f}\right)=\phi_{I}\left(t_{f}\right)=\phi_{J}\left(t_{f}\right)=\phi_{R}\left(t_{f}\right)=0$

On the interior of the control set and since $0 \leq a_{i} \leq u_{i}(t) \leq b_{i} \prec 1$ for $\mathrm{i}=1,2,3$ we obtain;

$$
\begin{aligned}
& 0=\frac{\partial H}{\partial u_{1}}=2 A_{1} u_{1}+\lambda S^{*}(t)\left[\phi_{S}-\phi_{E}\right] \\
& \Rightarrow 2 A_{1} u_{1}=\lambda S^{*}(t)\left[\phi_{E}-\phi_{S}\right] \\
& 0=\frac{\partial H}{\partial u_{2}}=2 A_{2} u_{2}+I^{*}(t) \phi_{I} \\
& \Rightarrow 2 A_{2} u_{2}=-I^{*}(t) \phi_{I} \\
& 0=\frac{\partial H}{\partial u_{3}}=2 A_{3} u_{3}+I^{*}(t) \phi_{I}+J^{*}(t) \phi_{J}-\left(I^{*}(t)+J^{*}(t)\right) \phi_{R} \\
& \Rightarrow 2 A_{3} u_{3}=\left(I^{*}(t)+J^{*}(t)\right) \phi_{R}-\left[I^{*}(t) \phi_{I}+J^{*}(t) \phi_{J}\right]
\end{aligned}
$$

Therefore,

$$
\begin{aligned}
& u_{1}^{*}=\max \left\{0, \min \left(1, \frac{\lambda S^{*}(t)\left[\phi_{E}-\phi_{S}\right]}{2 A_{1}}\right)\right\} \\
& u_{2}^{*}=\max \left\{0, \min \left(1, \frac{-I^{*}(t) \phi_{I}}{2 A_{2}}\right)\right\} \\
& u_{3}^{*}=\max \left\{0, \min \left(1, \frac{\left(I^{*}(t)+J^{*}(t)\right) \phi_{R}-\left(I^{*}(t) \phi_{I}+J^{*}(t) \phi_{J}\right)}{2 A_{3}}\right)\right\}
\end{aligned}
$$

By standard control arguments involving the bounds on the control variables, we have

$$
\begin{aligned}
& u_{1}^{*}=\left\{\frac{\lambda S^{*}(t)\left[\phi_{E}-\phi_{S}\right]}{2 A_{1}}, \quad \text { if } 0<\frac{\lambda S^{*}(t)\left[\phi_{E}-\phi_{S}\right]}{2 A_{1}}<1\right. \text {, } \\
& 0 \quad \text { if } \frac{\lambda S^{*}(t)\left[\phi_{E}-\phi_{S}\right]}{2 A_{1}} \leq 0 \\
& 1 \quad \text { if } \frac{\lambda S^{*}(t)\left[\phi_{E}-\phi_{S}\right]}{2 A_{1}} \geq 1
\end{aligned}
$$

Similarly, we have 


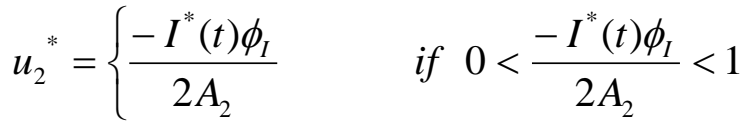

$$
\begin{aligned}
& 0 \quad \text { if } \frac{-I^{*}(t) \phi_{I}}{2 A_{2}} \leq 0 \\
& 1 \quad \text { if } \quad \frac{-I^{*}(t) \phi_{I}}{2 A_{2}} \geq 1
\end{aligned}
$$

Again, we also have

$$
\begin{gathered}
u_{3}^{*}= \begin{cases}\frac{\left(I^{*}(t)+J^{*}(t)\right) \phi_{R}-\left(I^{*}(t) \phi_{I}+J^{*}(t) \phi_{J}\right)}{2 A_{3}} & \text { if } 0<\frac{\left(I^{*}(t)+J^{*}(t)\right) \phi_{R}-\left(I^{*}(t) \phi_{I}+J^{*}(t) \phi_{J}\right)}{2 A_{3}}<1 \\
0 & \text { if } \frac{\left(I^{*}(t)+J^{*}(t)\right) \phi_{R}-\left(I^{*}(t) \phi_{I}+J^{*}(t) \phi_{J}\right)}{2 A_{3}} \leq 0 \\
1 & \text { if } \frac{\left(I^{*}(t)+J^{*}(t)\right) \phi_{R}-\left(I^{*}(t) \phi_{I}+J^{*}(t) \phi_{J}\right)}{2 A_{3}} \geq 1\end{cases}
\end{gathered}
$$

This completes the proof.

The optimality system consists of the state system coupled with the adjoint system with the initial and tranversality conditions together with the characterization of the optimal control pair.

$$
\begin{aligned}
& u_{1}^{*}=\max \left\{0, \min \left(1, \frac{\lambda S^{*}(t)\left[\phi_{E}-\phi_{S}\right]}{2 A_{1}}\right)\right\} \\
& u_{2}^{*}=\max \left\{0, \min \left(1, \frac{-I^{*}(t) \phi_{I}}{2 A_{2}}\right)\right\} \\
& u_{3}^{*}=\max \left\{0, \min \left(1, \frac{\left(I^{*}(t)+J^{*}(t)\right) \phi_{R}-\left(I^{*}(t) \phi_{I}+J^{*}(t) \phi_{J}\right)}{2 A_{3}}\right)\right\}
\end{aligned}
$$

Substituting (4.6) into (4.1) we obtained the following optimality system; 


$$
\begin{aligned}
\frac{d S(t)}{d t}= & \pi+\sigma R(t)-\left(1-\max \left\{0, \min \left(1, \frac{\lambda S^{*}(t)\left[\phi_{E}-\phi_{S}\right]}{2 A_{1}}\right)\right\}\right) \lambda S(t)-\mu S(t) \\
\frac{d E(t)}{d t}= & \left(1-\max \left\{0, \min \left(1, \frac{\lambda S^{*}(t)\left[\phi_{E}-\phi_{S}\right]}{2 A_{1}}\right)\right\}\right) \lambda S(t)-(\kappa+\mu) E(t) \\
\frac{d I(t)}{d t}= & \kappa E(t)-(\xi+\mu+\delta) I(t)-\left\{\left(\left(1-\max \left\{0, \min \left(1, \frac{-I^{*}(t) \phi_{I}}{2 A_{2}}\right)\right\}\right)\right.\right. \\
& \left.+\left(1-\max \left\{0, \min \left(1, \frac{\left(I^{*}(t)+J^{*}(t)\right) \phi_{R}-\left(I^{*}(t) \phi_{I}+J^{*}(t) \phi_{J}\right)}{2 A_{3}}\right)\right\}\right)\right\} I(t) \\
\frac{d J(t)}{d t}= & \xi I(t)-\left(\mu+\delta_{j}\right) J(t)-\left(1-\max \left\{0, \min \left(1, \frac{\left(I^{*}(t)+J^{*}(t)\right) \phi_{R}-\left(I^{*}(t) \phi_{I}+J^{*}(t) \phi_{J}\right)}{2 A_{3}}\right)\right\}\right) J(t) \\
\frac{d R(t)}{d t}= & \left(1-\max \left\{0, \min \left(1, \frac{\left(I^{*}(t)+J^{*}(t)\right) \phi_{R}-\left(I^{*}(t) \phi_{I}+J^{*}(t) \phi_{J}\right)}{2 A_{3}}\right)\right\}\right) I(t) \\
& +\left(1-\max \left\{0, \min \left(1, \frac{\left(I^{*}(t)+J^{*}(t)\right) \phi_{R}-\left(I^{*}(t) \phi_{I}+J^{*}(t) \phi_{J}\right)}{2 A_{3}}\right)\right\}\right) J(t)-\sigma R(t)-\mu R(t)
\end{aligned}
$$

Due to the prior boundedness of the state and adjoint functions and the resulting Lipschitz structure of the ordinary differential equations, we can obtain the uniqueness of the optimal control for small $t_{f}$, following technique from [24]. The uniqueness of the optimal control follows from the uniqueness of the optimality system (4.10). There is a restriction on the length of time interval in order to guarantee the uniqueness of the optimality system. This smallness restriction of the length on the time is due to the opposite time orientations of the optimality system; the state problem has initial values and the adjoint problem has final values. This restriction is very common in control problems (see[5,19]).

\section{NUMERICAL SIMULATIONS}

In order to verify the best control strategy to be adopted in the control of measles disease, the numerical simulations is analyzed by using finite differencing method using the following set of parameter values:

Table 2: Parameter value

\begin{tabular}{ll}
\hline Parameters & Values \\
\hline$\gamma_{1}$ & 0.8 \\
$\gamma_{2}$ & 0.8 \\
$\sigma$ & 0.2 \\
$\kappa$ & 0.09 \\
$\mu$ & 0.1 \\
$\pi$ & 2000 \\
$\delta$ & 0.1 \\
$\xi$ & 0.1 \\
$\eta$ & 0.01 \\
$\beta$ & 0.2 \\
\hline
\end{tabular}



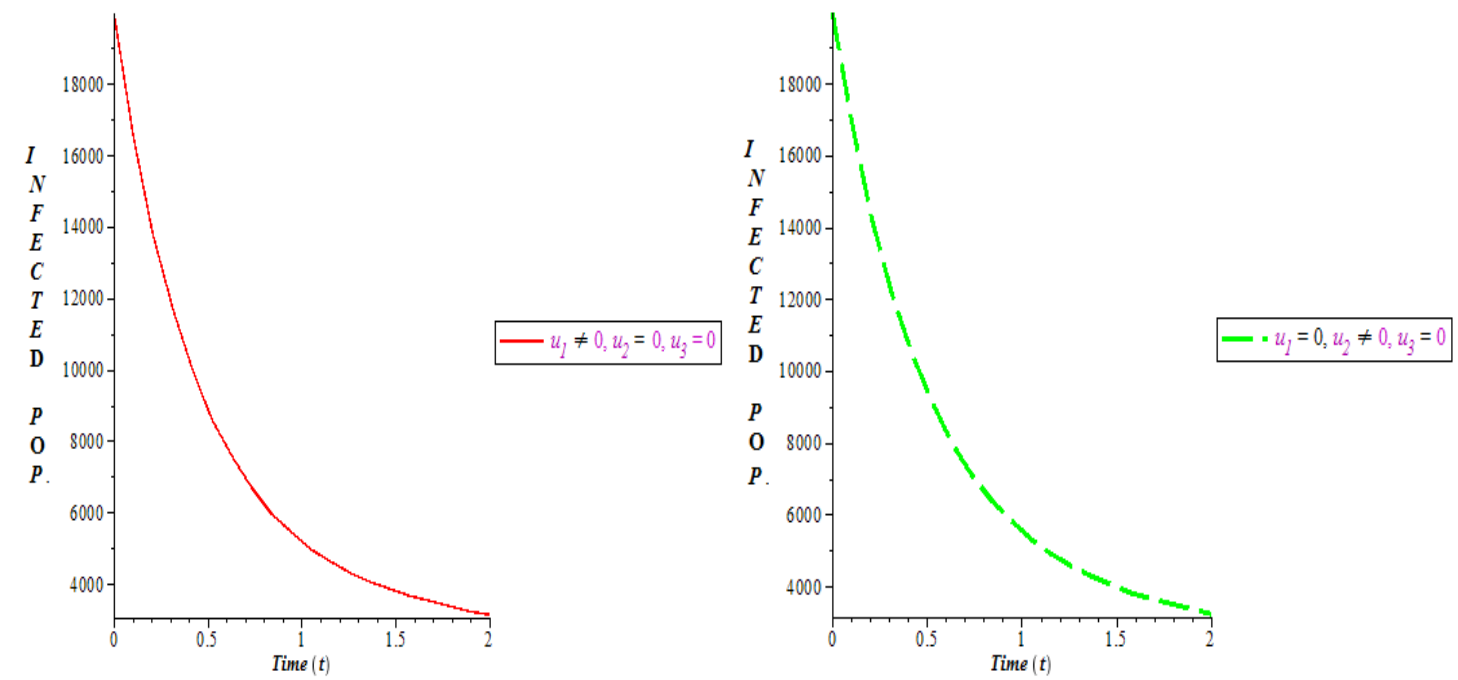

Figure 1: Optimal control graph for vaccine

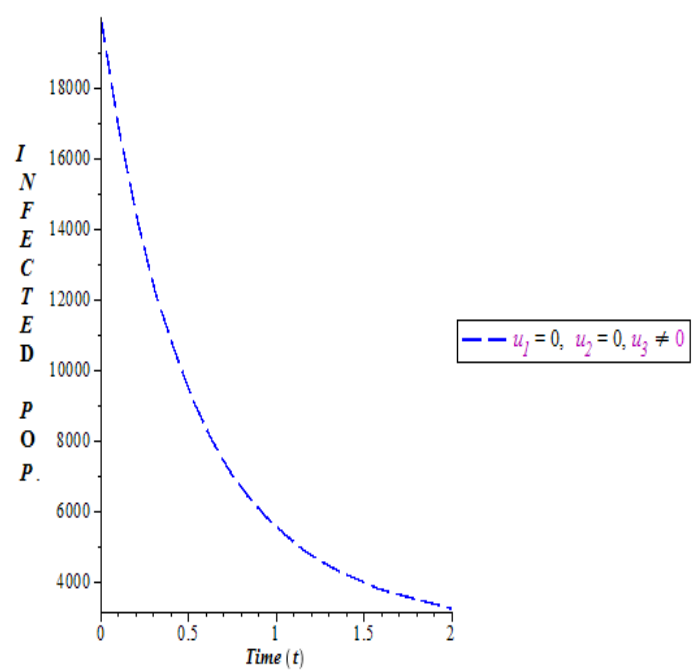

Figure 2: Optimal control graph for education

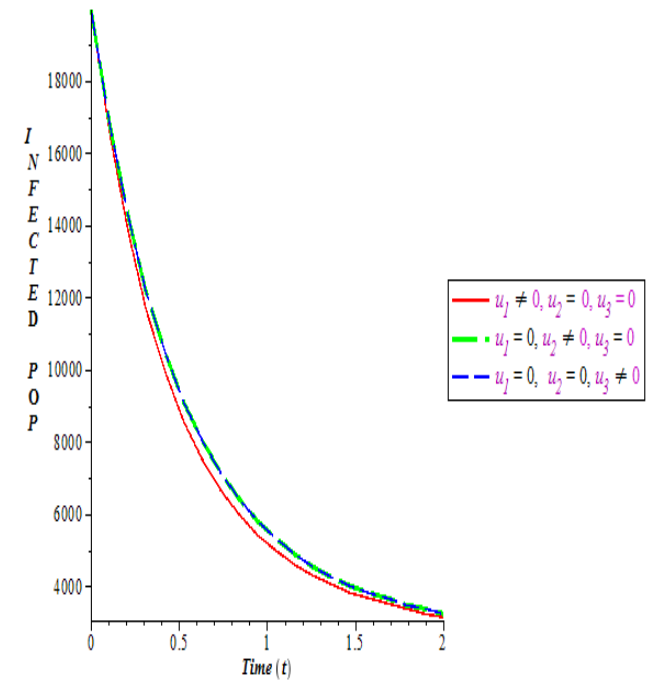

Figure 3: Optimal control graph for the efficacy of drug

Figure 4: Optimal control graph for the combination of three strategies

\section{DISCUSSION OF RESULTS}

We presented and analyzed five (5) compartmental models which incorporated isolated infected individual during treatment so as to gain insight into the measles infection transmission dynamics. It was shown that the disease free equilibrium is locally asymptotically stable whenever $R_{0}<1$ and unstable whenever $R_{0}>1$. We also showed that an endemic situation exists whenever $R_{0}>1$.

Figures 1, 2 and 3 show the optimal control graph of vaccine, education/campaign and efficacy of drug respectively. In each case, there is reduction in the number of measles infected individuals. Also, figure 4 shows combination of the three controls in order to know the best control. It was observed from figure 4 that vaccine has a pronounced effect on the control of 
measles as compared with other two controls, in the sense that fewer people would be infected if vaccine is administered.

\section{CONCLUSION}

The results above show that vaccine control strategy yields a better result over treatment of the disease and Education/campaigning, which implies that administering vaccine efficiently plays a vital role in reducing dynamical spread of measles. Vaccination of susceptible individuals reduces the dynamical spread of measles in the environment, thereby reducing those that will be infected. In conclusion, timely given of vaccine should be adopted as best control strategy against the dynamical spread of measles.

\section{REFERENCES}

[1] Adeoye I.A, Dairo M.D, Adekunle L.V, Adedokun H.O, and Makanjuola J. (2010): Investigation of a measles outbreak in a Rural Nigerian community - The Aladura experience. African Journal of Microbiology Research Vol. 4(5), pp. 360-366.

[2] Adewale S.O, Podder C.N, and Gumel A.B (2009): Mathematical Analysis of a TB Transmission Model with DOTS. Canadian Applied Mathematical Quarterly Volume 17, number 1, Spring 2009.

[3] Adewale S.O, Olanrewaju P.O, Taiwo S.S, Anake T.A and Famewo,M.M (2012) Mathematical Analysis of the effect of Immunization on the dynamical spread of Measles. International Electronic Journal of Pure \& Applied Maths.

[4] Adewale S.O, Mohammed I.T and Olopade I.A (2014) Mathematical Analysis of Effect of Area on the Dynamical Spread of Measles. International Organization of Scientific Research.

[5] Agusto F.B, Marcus N, Okosun K.O (2012): Application of Optimal Control To The Epidemiology of Malaria. Electronic Jour. Of Diff. Eqns, Vol 81, Pp 1-22

[6] Annual Report of the National Disease Surveillance Centre,2000. Dublin, Health Protection Surveillance Centre; 2001. ISSN:1649-0436. Available from: http://www.hpsc.ie/hpsc/ AboutHPSC/AnnualReports/File, 520, en.pdf.

[7] Ca'ceres VM, Strebel PM, Sutter $R W$ (2000): Factors determining prevalence of maternal antibody to measles virus throughout infancy: a review. Clin Infect Dis 2000;31: 110-19.

[8] Carabin H, Edmunds WJ, Kou U, van den Hof S \& Nguyen VH (2002). The average cost of measles cases and adverse events following vaccination in industrialised countries. BMC Public Health 2:22 (http://www.biomedcentral.com/1471-2458/2/22).

[9] Driessche P. van den and Watmough J.(2002): Reproduction numbers and sub-threshold endemic equilibria for compartmental models of disease transmission, Math.Biosci. 180 (2002), 29\{48\}.

[10] Fleming W, and Rishel R(1975): Deterministic and Stochastic Optimal Control. Springer Verlag, New York

[11] Grais RF, Dubray C, Gerstl S (2007): Unacceptably high mortality related to measles epidemics in Niger, Nigeria, and Chad. PLoS Med 2007;4:el6.

[12] Grais R.F, Ferrari M.J, Dubray C, Bjornstad O.N, Grenfell B.T, Djibo A, Fermon, F, Guerin P.J (2006): Estimating Transmission intensity for a measles epidemic in Niamey, 
Niger: Lessons for intervention. Transactions of Royal Society of Tropical Medicine and Hygiene (2006)100,867-873.

[13] Hattaf. K, and Yousfi. N (2011): Dynamics of HIV infection Model with Therapy and cure rate. International Journal of Tomography and Statistics, 16(11), 74-80.

[14] Hattaf. K, and Yousfi. N (2012): Two Optimal Treatments of HIV Infection Model. World Journal of Modelling and Simulation, Vol. 8(2012), No.1, Pp. 27-35

[15] Hethcote H.W. and Waltman P. (1973):Optimal vaccination schedules in a deterministic epidemic model.Math. Biosci. 18 (1973), 365-382.

[16] Hethcote H.W. and Thieme H. R.(1985): Stability of the endemic equilibrium in epidemic models with subpopulations, Math. Biosci. 75 (1985), 205\{227\}.

[17] Hethcote H.W. (1989): Optimal ages for vaccination for measles. Math. Biosci. 89 (1989), $29-52$.

[18] Hethcote H.W., (2000): The mathematics of infectious diseases. Society for Industrial and Applied Mathematics Siam Review, Vol. 42, No. 4, pp. 599-653.

[19] Joshi, H. R. (2002): Optimal Control of an HIV Immunology Model, Optim. Control Appl. Math, 23(2002), 199-213.

[20] Lakshmikantham V, Leela S, and Martynyuk A. A. (1989). Stability analysis of nonlinear systems.Marcel Dekker, Inc., New York and Basel.

[21] Lara J Wolfson, Rebecca F Grais,Francisco J Luquero, Maureen E Birmingham and Peter M Strebel (2009): Estimates of measles case fatality ratios: a comprehensive review of community-based studies. International Journal of Epidemiology 2009;38:192205doi:10.1093/ije/dyn224.

[22] Lukes,D.L (1982): Differential Equation: Classical to Controlled. Academic Press, New york

[23] Ousmane MOUSSA TESSA (2006): Mathematical model for control of measles by vaccination.MSAS Symposium 2006.

[24] Pontryagin, L.S; Boltyanski, V.G; Gamkrelidze, R.V; Mishchenko, E.F (1962): The Mathematical Theory of optimal processes, Wiley, New York, 1962.

[25] Ramsay M, Gay N, Miller E, White J, Morgan-Capner P \& Brown D (1994). The epidemiology of measles in England and Wales: Rationale for the 1994 national vaccination.

[26] Roberts M.G and Tobias M.I (2000): Predicting and Preventing Measles epidemics in New Zealand: application of a mathematical model. Epidemiol. Infect. (124) 279-287.

[27] Spotlight on Measles 2010: Measles outbreak in Ireland 2009-2010. BMC Public Health 2:22 (http://www.biomedcentral.com/1471-2458/2/22)

[28] Strebel P, Cochi S, Grabowsky M (2003): The unfinished measles immunization agenda. J Infect Dis 2003;187 (Suppl 1):S1-S7.

[29] World Health Organization, (2004): Measles vaccines: WHO position paper. Wkly. Epidemiol. Rec. 79, 130-142.

[30] World Health Organization (2009): Measles Fact Sheet No 286. Geneva Switzerland: www, who, int/mediacentre/factsheets/fs286/en.

[31] World Health Organization(2011): WHO Vaccine Preventable Diseases: Monitoring System2011 Global Summary, available at:http://www.who.int/immunization_monitoring/en/globalsummary/timeseries/tsincidenc emea.htm. 